\title{
Le marimbula, un lamellophone africain aux Antilles néerlandaises
}

Jos Gansemans

\section{(2) OpenEdition}

\section{Journals}

Édition électronique

URL : http://journals.openedition.org/ethnomusicologie/2334

ISSN : 2235-7688

Éditeur

ADEM - Ateliers d'ethnomusicologie

Édition imprimée

Date de publication : 1 janvier 1989

Pagination : 125-132

ISBN : 2-8257-0178-5

ISSN : 1662-372X

\section{Référence électronique}

Jos Gansemans, «Le marimbula, un lamellophone africain aux Antilles néerlandaises », Cahiers

d'ethnomusicologie [En ligne], 2 | 1989, mis en ligne le 15 septembre 2011, consulté le 01 mai 2019.

URL : http://journals.openedition.org/ethnomusicologie/2334 


\title{
LE MARIMBULA, UN LAMELLOPHONE AFRICAIN AUX ANTILLES NÉERLANDAISES
}

\author{
Jos Gansemans
}

Situées à une trentaine de kilomètres de la côte septentrionale du Venezuela, les îles Aruba, Bonaire et Curaçao font partie des Antilles néerlandaises. De même que les autres îles de la mer des Caraïbes, elles ont connu un passé turbulent dès 1499, année de leur découverte par les Espagnols qui les occupèrent jusqu'en 1643 et en soumirent les habitants autochtones, les Caiquetios appartenant au groupe linguistique Arawak et habitant également le nord du Venezuela. En 1643, les trois îles furent conquises par les Hollandais qui les administrent jusqu'à aujourd'hui, sans compter une breve présence anglaise de 1799 à 1816 .

Dépourvues de richesses minières, Aruba, Bonaire et Curaçao n'avaient aucun intérêt pour les Espagnols qui déportèrent la plupart des hommes indigènes vers d'autres îles (Haïti, Saint-Domingue) pour les faire travailler dans les mines d'or, et affectèrent ceux qui restaient au travail dans les plantations et à l'élevage.

La conquête par les Hollandais et l'implantation de la West-Indische Compagnie (Compagnie des Indes occidentales) amenèrent un tournant dans l'évolution culturelle et économique des trois îles, à travers la traite des esclaves noirs embarqués en Afrique à St. George d'Elmina (Ghana) et à Luanda (Angola) notamment. Ainsi se propagèrent des pratiques culturelles d'Afrique occidentale et centrale dans des domaines aussi divers que la langue, la religion, le système de parenté, la nourriture, la tradition orale, la musique et la danse. Or, Curaçao fonctionnant jadis comme port de transition pour les esclaves à destination d'autres îles des Caraibes (Cuba, Haïti, Saint-Domingue, Jamaïque, Trinidad), ce n'est qu'une minorité d'esclaves, et certainement pas les plus résistants physiquement, qui y demeurèrent. Il en résulta une très grande diversité de groupes ethniques, tous relativement peu nombreux.

Contrairement aux autres îles, où le nombre d'esclaves originaires du même groupe était beaucoup plus élevé, le maintien d'une tradition ethnique particulière s'est avéré quasi impossible en Aruba, à Bonaire et à Curaçao. Dans le domaine de la culture musicale, on constate ainsi que certains instruments de 
musique se limitent à telle ou telle des trois îles, comme c'est le cas de l'arc à bouche benta, du racleur wiri, de la trompe cachu, du tambour d'eau bastel, de la clarinette idioglotte beku, du tambour à peau tambu, des tubes pilonnants bamba et de quelques instruments de percussion (chapi, agan). La culture musicale des îles en question est donc plutôt constituée d'un amalgame d'éléments fragmentaires provenant tant du Golfe de Guinée (Ghana, Bénin, Nigeria, Togo) que de la côte atlantique de l'Afrique centrale (Angola). C'est également pour cette raison qu'un instrument proprement africain comme le lamellophone marimbula, pourtant présent à Cuba depuis longtemps, n'est apparu dans les îles qu'au début du $\mathrm{XX}^{\mathrm{e}}$ siècle, à la suite de la migration temporaire d'une partie de leur population.

L'économie des îles étant peu développée et basée surtout sur le commerce (Curaçao), les plantations à dimensions réduites (Aruba et Bonaire) et la production de sel (Bonaire), la situation sociale et économique des Noirs changea peu, même après l'abolition de l'esclavage en 1863. En effet, la plupart des habitants restèrent économiquement dépendants de leur maître, shon. D'autres choisirent l'émigration temporaire ou définitive vers d'autres îles pour y travailler dans les plantations, comme ce fut le cas des Arubains et Bonairiens qui partirent pour Cuba. Quant aux Bonairiens, marins réputés qui sillonnaient la mer des Caraibes à la fin du $\mathrm{XIX}^{\mathrm{e}}$ et au début du $\mathrm{XX}^{\mathrm{e}}$ siècle, ils avaient gardé des liens avec les peuples noirs et leurs cultures musicales traditionnelles. C'est eux qui découvrirent le lamellophone marimbula à Cuba et l'incorporèrent à leurs propres ensembles musicaux. Comme nous l'ont confirmé plusieurs informateurs-musiciens, le marimbula aurait été introduit en Aruba vers 1922, au moment où les travailleurs rentraient de Cuba, et presque à la même époque (1925-1930) à Bonaire, aux dires de musiciens âgés qui se souviennent encore de la première apparition de l'instrument dans les ensembles populaires.

Bien que le marimbula ne subsiste actuellement que dans les ensembles musicaux de Bonaire et d'Aruba, il existait aussi autrefois à Curaçao, comme en témoignent les enregistrements réalisés entre 1954 et 1962 par P. Brenneker et E. Juliana (Collection Zikinza No 1166, 1284-1289, 1298, 1299). Ajoutons à ce propos que Van Wengen (1966: 33) signale un instrument appelé tambora à Curaçao, qui était d'après lui identique au marimbula et servait de basse dans les ensembles dits "típicos» qui exécutaient la musique de danse populaire. Mais depuis 1970 environ, ce dernier instrument a cédé la place à la guitare basse électrique.

Bennett (1976: 10) mentionne pour Aruba plusieurs synonymes du nom de marimbula, à savoir: marimba, manimba ou marimula. A Bonaire, la dénomination marimbula est courante.

Étymologiquement, le terme de marimbula renvoie au radical bantou imba- qui se rapporte à tout ce qui a trait au chant ou à la mélodie. Le terme de ma-dimba ou marimba étant le pluriel de di-dimba, soit "touche (d'un xylophone)», il peut donc se traduire par «les touches». Dans d'autres langues bantoues, on indique par ce même nom les lamelles ou les touches du lamellophone. Toujours en bantou, le suffixe $-u l(a)$ ou $-i l(a)$ indique que l'on fait 
quelque chose pour quelqu'un. Par exemple, kwimba signifie «chanter», et kwimbila, «chanter en l'honneur de quelqu'un». Marimbula pourrait donc signifier littéralement «l'instrument qui joue (chante) pour quelqu'un».

La première illustration du lamellophone est fournie par Bonanni (1722: pl. 174) qui l'appelle marimba de Cafri, alors que sa première mention remonte à 1586 lorsque Dos Santos évoque sous le nom de ambira un lamellophone à neuf touches métalliques (Kirby 1953: 66). Sa zone de diffusion s'étend actuellement de l'Afrique du Sud et de l'est du Mozambique et de l'Afrique centrale à l'ouest du Niger, d'où il suivit la voie de la traite jusque dans les Caraibes (Söderberg 1956: 112-117). Les différents types de lamellophones que l'on retrouve dans les îles reflètent leur distribution en Afrique subsaharienne: on en rencontre en Afrique occidentale, chez les Nago du Bénin (aguidigbo) et les Peul du Cameroun $(\operatorname{sen} z a)$ notamment.

A Cuba, il a été signalé en 1839 par Pichardo dans son Diccionario provincial casi razonado de voces cubanas. Un siècle plus tard, l'instrument est décrit ainsi par Castellanos (1927: 2-4):

"La marimba afrocubana, a veces llamada marimbula, generalmente es un pequeno envase de madera de $44 \mathrm{cms}$ de largo, $19 \mathrm{cms}$ de alto, $26 \mathrm{cms}$ de ancho».

Mais c'est à Ortiz que nous devons un aperçu complet des différents types de marimbula présents à Cuba. Comme en Afrique subsaharienne, où il portait les noms de kembe, sanji, mbira et leurs variantes, il se distinguait par sa forme, ses dimensions, les matériaux utilisés pour sa fabrication et le nombre de lamelles, suivant les ethnies qui en jouaient. La boîte rectangulaire aux dimensions réduites du marimbula cubain rappelle celle du lamellophone dit «fluvial» largement répandu au Zaïre, le type d'instrument joué par les Kongo (Zaïre et Angola) étant le plus proche de l'ancien marimbula de Cuba.

$\mathrm{Au}$ Venezuela, par contre, l'instrument en question est peu répandu. Pollack-Eltz (1978: 29) signale un type de marimbula dans la province de Miranda, où vivent principalement des descendants d'esclaves. Pourvu de trois lamelles, cet instrument mesure $40 \mathrm{~cm}$ de haut, $32 \mathrm{~cm}$ de large, et sa profondeur est de $20 \mathrm{~cm}$. L'auteur caractérise sa fonction musicale comme suit:

"The instrument is used to accompany secular music and never appears in folk-religious fiestas, as for example the celebration in honor of San Juan. It accompanies dances held on Saturday nights in the villages and is used together with the tumbadora drum, one or more cuatros, some rattles and a charrasca (rasp). The music played is merengue-style, popular in the Carribean area».

List et Schechter (1984: 617) signalent que le marimbula est joué sur la côte atlantique et pacifique de la Colombie comme instrument de basse. Les lamelles y sont faites de suncho, de segments de bandes métalliques tendues autour 
de caisses en bois. Thompson (1975-76: 142-145) donne une description détaillée du marimbula de Puerto Rico, et il ajoute que ce lamellophone largement répandu dans l'île y est apparu vers 1852 . D'autre part, il cite la pièce de théâtre de Ramón Cabbalero, La juega de gallos o El negro bozal, dans laquelle le protagoniste, José, amuse la créole Nazaria par le jeu du marimba.

La facture, les dimensions et la forme du marimbula sont presque identiques à travers toutes les Caraibes. L'instrument est fabriqué par les musiciens eux-mêmes, ce qui explique sa facture parfois rudimentaire. Selon les coutumes locales ainsi que l'habileté du luthier, la forme de la caisse de résonance est rectangulaire, carrée ou parallélogramme (fig. 1-3). Les faces frontale et dorsale de l'instrument sont en contreplaqué, tandis que les faces inférieure, latérales et supérieure sont faites de planches de bois plus épaisses.

Il est à noter que la table d'harmonie de chaque instrument est pourvue d'une ouïe sous la partie vibrante des lamelles.

Les dimensions des marimbula des îles Aruba (relevées à Alto Vista et Oranjestad) et Bonaire (relevées à Nikiboko et Rincon) sont similaires: leur hauteur varie entre 35 et $50 \mathrm{~cm}$, leur largeur, entre 54 et $60 \mathrm{~cm}$, et leur profondeur, entre 20 et $27 \mathrm{~cm}$. Tous les instruments vus, sauf un, étaient munis de six touches.

Les lamelles lenga, traduction littérale de «langues», déterminent la sonorité de l'instrument. Il est remarquable que leur matériau, à savoir des ressorts de vieux phonographes, soit identique dans toute la région des Caraibes, car cette matière première est actuellement difficile à trouver. Maintenant, on se sert plutôt des ressorts d'horloge à pendule, bien que ceux-ci soient plus minces et ne produisent pas le son grave typique des lamelles larges. Précisons que la largeur des lamelles peut varier entre 25 et $32 \mathrm{~mm}$. Un autre matériau typique est la bande de fer avec laquelle on cercle les caisses ou les tonneaux en bois. Dans un seul cas, le musicien avait utilisé les rubans d'une vieille scie après en avoir limé les dents. Comme il arrive fréquemment en organologie populaire, la matière première pour la fabrication des lamelles est du matériel de récupération.

D'ordinaire, les lamelles sont fixées sur la caisse de résonance au moyen de deux baguettes superposées, entre lesquelles elles sont serrées à l'aide d'un vis ou d'un écrou à ailettes permettant de réaccorder les touches en les dévissant, de sorte que la partie vibrante de la lamelle puisse être raccourcie (un ton plus haut) ou rallongée (un ton plus bas).

Dans les îles en question, le nombre des lamelles varie entre cinq et sept, tandis qu'ailleurs, le marimbula est muni, soit de trois ou quatre touches, comme à Haïti et à Saint-Domingue, soit de dix ou plus, comme à Cuba et à Puerto Rico. D'après l'informateur-musicien Cola (Antriol, Bonaire), il existait dans le passé des marimbula à douze touches, qui étaient probablement calqués entièrement sur les instruments cubains. En tenant compte de la fonction musicale de l'instrument, qui est harmonique et non mélodique dans la musique populaire de Bonaire et d'Aruba, il est fort probable que le nombre des touches ait diminué depuis lors. 
Fig. 1: Marimbula à six touches. Rincon (Bonaire). Archives du Musée royal de l'Afrique centrale - Ethnomusicologie $\mathrm{N}^{\circ} 6100$.

Fig. 2: Marimbula à cinq touches. Alto Vista (Aruba). Archives du Musée royal de l'Afrique centrale - Ethnomusicologie $N^{\circ} 6097$.

Fig. 3: Marimbula en forme de parallélogramme. Rincon (Bonaire). Archives du Musée royal de l'Afrique centrale - Ethnomusicologie $N^{\circ}$ 5195. Dessins: Soheil Azzam, sur la base de photos de J. Gansemans, 1983-84.
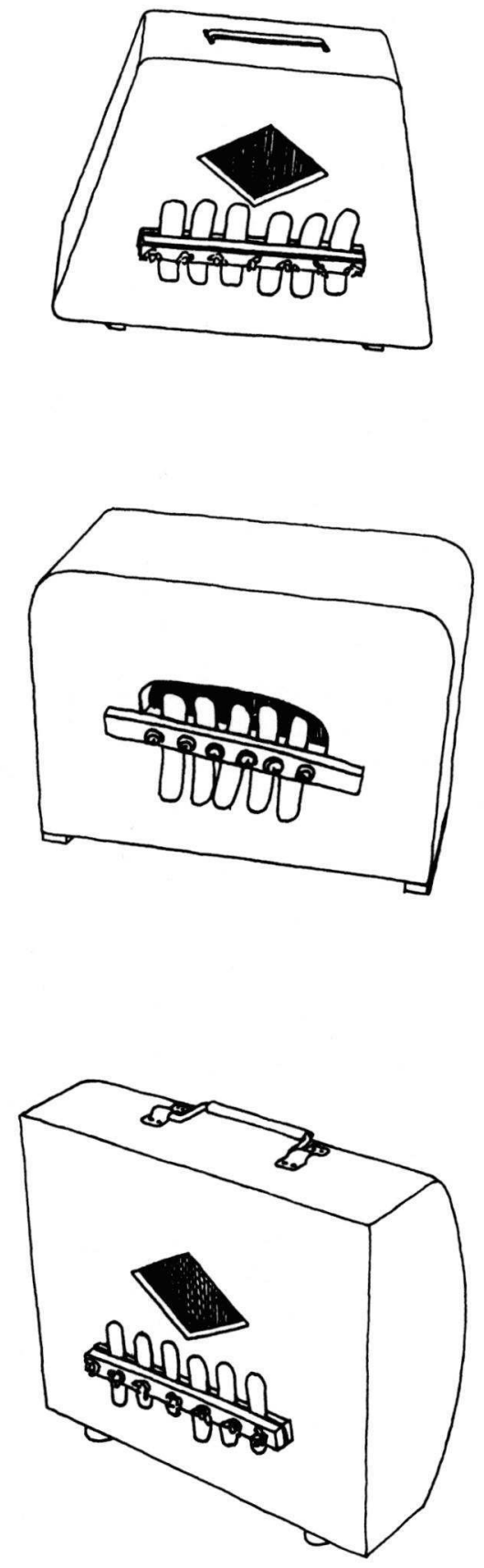
Comme nous l'avons déjà précisé, le marimbula sert à produire une basse ostinato. Par conséquent, l'accord des lamelles se fait selon la tonalité du chant qu'il accompagne, et il reprend souvent celui de la guitare ou de la kuarta, deux instruments voués à l'accompagnement harmonique du chant.

Souvent, le musicien n'accorde pas toutes les lenga du marimbula, mais se contente de l'accord des trois ou quatre lamelles qu'il pincera effectivement, les autres restant muettes. C'est pourquoi il est souvent inutile de transcrire toutes les notes du marimbula, puisqu'elles ne représentent la tonalité que partiellement. Lors de notre enquête sur le terrain, nous avons transcrit plusieurs tonalités:

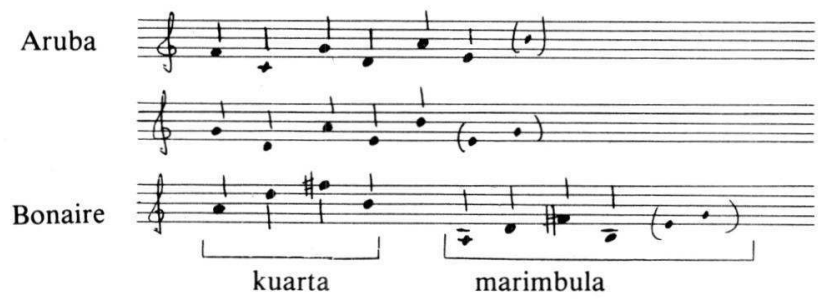

La fonction musicale du marimbula se limitant principalement au jeu des ostinati, il représente la base harmonique de la musique populaire, mais il forme aussi avec le racleur wiri et le tambour bari, la base rythmique de l'ensemble musical. Les deux fonctions - rythmique et harmonique - du marimbula sont typiques de la musique de danse des Caraibes, qui plonge aussi ses racines dans la musique ibérique. Par ses caractéristiques musicales, le marimbula présente donc une conjonction d'éléments africains et européens qui assurent la spécificité de la musique populaire de la région.

La position de jeu du marimbula est verticale. Le musicien le pose sur le sol (fig. 4) et s'assied dessus, les jambes écartées. Parfois, il le pose sur une chaise et le laisse reposer contre son ventre (fig. 5). A Bonaire, les informateurs nous ont signalé que le marimbula était également porté lors des cortèges. Dans ce cas, il pendait horizontalement au cou du musicien à l'aide d'une bande de cuir.

Les touches sont pincées avec le médius et l'index, tandis que le musicien frappe un rythme supplémentaire avec les pouces sur la caisse de résonance. Parfois, seules deux (la fondamentale et la dominante) ou trois touches sont jouées avec une main, l'autre main servant ainsi à battre le rythme qui retient parfois davantage l'attention du public que le motif en ostinato du marimbula.

L'ordre des lenga et l'échelle qui en résulte sont déterminés par le nombre de touches. Pour les instruments à trois ou quatre touches, il s'agit d'une série tonale ascendante. Pour les instruments à cinq touches et plus, on préfère mettre la touche la plus basse au milieu, en allant alternativement vers les deux extrémités aux sons aigus. Cet emplacement des touches est conforme à celui des lamellophones d'Afrique centrale appartenant au type «fluvial» (Laurenty 1962: 23). On le retrouve sur un spécimen arubain où trois touches sont pincées avec l'index gauche et quatre avec l'index droit.

Il est également typique que le marimbula soit absent des ensembles musicaux des centres urbains, alors qu'il fait partie intégrante de ceux des villages 


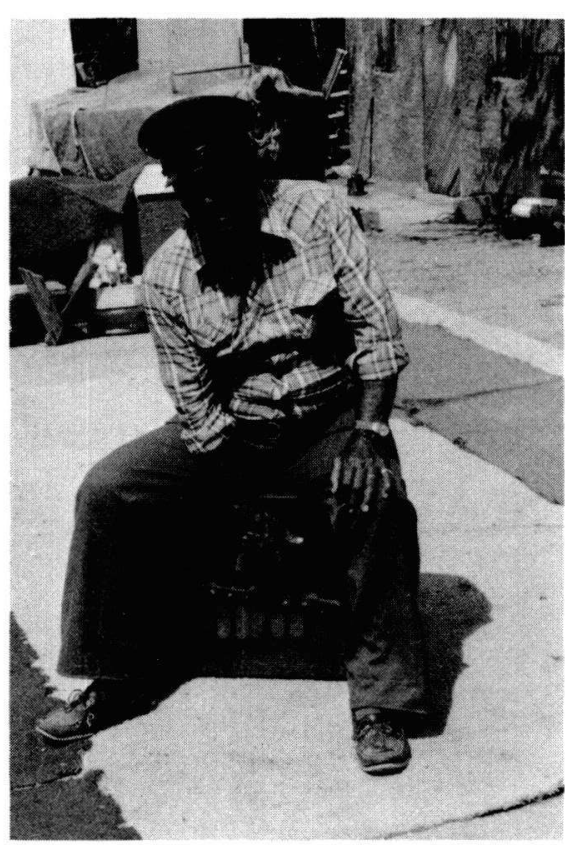

Fig. 4: Position de jeu habituelle. Tony Werlemann. Alto Vista (Aruba). Archives du Musée royal de l'Afrique centrale - Ethnomusicologie $\mathrm{N}^{\circ}$ 6095. Photo: J. Gansemans, 1983-84.

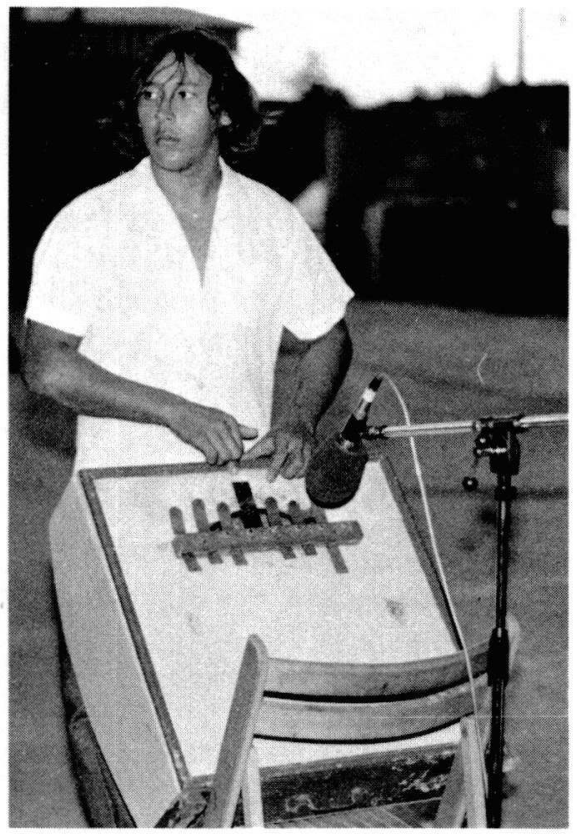

Fig. 5: Position de jeu. Montana (Aruba). Archives du Musée royal de l'Afrique centrale - Ethnomusicologie $N^{\circ}$ 5011. Photo: J. Gansemans, 1983-84.

où il apparaît habituellement dans les ensembles avec kuarta, mandoline, guitare et wiri (Bonaire) ou sinfonia di man (accordéon), bari, guitare et wiri (Aruba).

Lors des festivités, ces ensembles accompagnent de préférence les chants populaires, ainsi que la musique de danse (valse, rumba et polka).

Le musicien R. Keller, de Nikiboko (Bonaire), signalait à ce propos que «tous les conhunto dans les années quarante disposaient d'un marimbula pour accompagner la musique de fête et de mariage. Les gens aimaient pareil accompagnement qui jouait les rythmes de base». L'acquisition d'une contrebasse, instrument de basse préféré, étant dans la plupart des cas impossible, le marimbula était ironiquement dénommé poor man's bass des Caraibes.

Par contre, il est rarement présent dans les ensembles musicaux accompagnant les fêtes coutumières, comme celles du simadan ${ }^{1}$, de San Juan-San

Simidan: fête qui clôt la récolte de sorgho à Bonaire. 
Pedro $^{2}$, du dande $e^{3}$ ou du dera gai $i^{4}$. Cela prouve que le marimbula est d'importation récente sur les îles d'Aruba et de Bonaire et qu'il n'a jamais pu s'imposer dans les genres musicaux traditionnels, qui ne possèdent pas d'instrument de basse. L'incorporation du marimbula aux ensembles populaires est par conséquent un complément apprécié.

Ainsi le marimbula, actuellement joué de préférence par la population noire, se range-t-il parmi les instruments de musique d'origine africaine, tout en se situant, de par sa fonction musicale, dans un genre de musique de danse qui est dominé par la kuarta et la guitare et se rapproche de ce fait au style musical ibérique.

\section{Bibliographie}

BENNETT E.A.

1976 «Instrumenten». Skol \& Komunidat VII(1): 7-10.

BONANNI F.

1722 Descriptioni degli istrumenti armonici. Roma.

CASTELLANOS $\mathbf{J}$.

1927 Los instrumentos musicales de los Afrocubanos. Habana.

KIRBY P.

1953 The musical instruments of the native races of South Africa. Johannesburg: Witwatersrand University Press.

LAURENTY J.S.

1962 Les sanza du Congo. Tervuren.

LIST G. \& SCHECHTER G.M.

1984 «Marimbula». In: New Grove Dictionary of Musical Instruments. Ed. St. Sadie. London: MacMillan Press, vol. II: 616-17.

ORTIZ F.

1952 Los instrumentos de la música afrocubana. Habana: Ministerio de Educación.

POLLACK-ELTZ A.

1978 "The marimbula. An Afro-Américan Instrument». Review of Ethnology V(4): 28-30.

SÖDERBERG B.

1956 Les instruments de musique au Bas-Congo et dans les régions avoisinantes. Stockholm: The Ethnographical Museum of Sweden.

THOMPSON D.

1975- "A new world mbira: the Carribbean marimbula». African Music V(4): 140-148.

1976

VAN WENGEN G.D.

1966 «Muziekinstrumenten van de Antillen». Verre Naasten Naderbij I(1): 25-33.

2 San Juan-San Pedro: les 24 et 28 juin, les hommes qui répondent au prénom de Juan ou de Pedro reçoivent à la maison la visite d'un groupe de musiciens et de chanteurs en l'honneur de leur fête patronymique.

3 dande: à partir de minuit à la Saint-Sylvestre, un groupe de musiciens se déplace d'une mainson à l'autre pour transmettre en musique des voeux de Nouvel-An.

4 dera gai (litt. "enterrer le coq"): coutume fêtée le 24 juin (fête de St. Jean-Baptiste) qui culmine par le décapitement du coq enterré. 